\title{
Geochemistry of the Neoproterozoic Volcanic Rocks of the Nakora Area of Malani Igneous Suite, Barmer District, Western Rajasthan, India
}

\author{
Naresh Kumar*, Sarita Mann, Swati Rana, Savita Kumari, Yashpal, Paryant Ashwani \\ Department of Geology, Kurukshetra University, Kurukshetra, Haryana, India \\ Email: ${ }^{\star}$ sagwalnaresh@gmail.com
}

How to cite this paper: Kumar, N., Mann, S., Rana, S., Kumari, S., Yashpal and Ashwani, P. (2022) Geochemistry of the Neoproterozoic Volcanic Rocks of the Nakora Area of Malani Igneous Suite, Barmer District, Western Rajasthan, India. Open Journal of Geology, 12, 91-110.

https://doi.org/10.4236/oig.2022.122005

Received: January 12, 2022

Accepted: February 15, 2022

Published: February 18, 2022

Copyright $\odot 2022$ by author(s) and Scientific Research Publishing Inc. This work is licensed under the Creative Commons Attribution International License (CC BY 4.0).

http://creativecommons.org/licenses/by/4.0/ (c) (i) Open Access

\begin{abstract}
The Neoproterozoic rocks of the Nakora Ring Complex (NRC) consist of three phases (Extrusive, Intrusive and Dyke) that are based on the detailed geological mapping (contact relationship, mode of occurrence, position of xenolith, flows, dykes and veins) and their stratigraphic position. NRC consists mainly of acid volcanic rocks besides minor amount of basic rocks with intermediate calc-alkaline to tholeiitic affinities and occurs in the form of ring structures. The Nakora basaltic rocks show LREE enriched nature and they have consistent negative $\mathrm{Nb}, \mathrm{Ta}, \mathrm{Sr}$ and $\mathrm{Zr}$ anomalies. The HREE pattern is showing parallel arrangement with HREE pattern of other basic rocks. The Nakora acid volcanic rocks exhibit high LREE enrichment than the HREE with negative $\mathrm{Ba}, \mathrm{Sr}, \mathrm{Eu}$ and $\mathrm{Ti}$ anomalies in primitive mantle normalized multi-element diagrams. All the samples show negative $\mathrm{Ba}, \mathrm{Sr}, \mathrm{Eu}$ and $\mathrm{Ti}$ anomalies. The diminution in amounts of $\mathrm{Sr}$ and $\mathrm{Eu}$ is apparently related to the fractionation of feldspars or their retention in the refractory minerals resistant to melting in the lower crust. As compared to trachytes, the rhyolites show high $\mathrm{SiO}_{2}$, high $\mathrm{Al}_{2} \mathrm{O}_{3}$, low total alkalis, low total iron, low $\mathrm{TiO}_{2}$, high $\mathrm{CaO}$ and high $\mathrm{MgO}$. The petro-mineralogical and geo-chemical data specifies that the NRC rocks are generated from a co-magmatic source through a co-genetic process in a rift tectonic context. Petrogenetic modeling indicates that both the basic rocks and acid volcanic rocks of Nakora may have been derived from rocks akin to Bhilwara mafic metavolcanic/mixed Nakora gabbros and Siwana rhyolite/banded gneiss from Kolar Schist Belt by different degrees of partial melting respectively.
\end{abstract}

\section{Keywords}

Neoproterozoic, Volcanic Rocks, Geochemistry, Nakora, Malani Igneous Suite 


\section{Introduction}

The rock exposure revealed in the Nakora Ring Complex (NRC) is from the Trans-Aravalli Block's (TAB) Neoproterozoic Malani Igneous Suite (MIS). TAB is unique in the geological evolution of Indian Shield and Nakora area is located Northwestern part of the Indian Peninsular Shield. MIS is the Peninsular India's greatest A-type, anorogenic, high heat producing (HHP) acid magmatism (55, $000 \mathrm{~km}^{2}, 732 \mathrm{Ma}$ ) owing its origin to hot spot tectonics (Figure 1) (Kochhar 1973 [1]; Pareek 1981 [2]; Kochhar 1984 [3]; Bhushan 1985 [4]; Bhushan and Mohanty 1988 [5]; Vallinayagam 1988 [6]; Bhushan 1989 [7]; Eby and Kochhar 1990 [8]; Baskar 1992 [9]; Kochhar and Dhar 1993 [10]; Baskar and Sharma 1994 [11]; Sharma 1994 [12]; Kochhar et al. 1995 [13]; Dhar et al. 1996 [14]; Vallinayagam 1997 [15]; Bhushan and Chittora 1999 [16]; Vallinayagam 1999 [17]; Bhushan 2000 [18]; Pandit et al. 2001 [19]; Torsvic et al. 2001 [20]; Vallinayagam 2001 [21]; Bhushan and Chandrasekaran 2002 [22]; Kochhar 2004 [23]; Singh and Vallinayagam 2004 [24]; Vallinayagam 2004a [25]; Bhushan and Chittora 2005 [26]; Singh and Vallinayagam 2006 [27]; Vallinayagam and Kumar 2007 [28], Vallinayagam and Kumar 2008 [29]; Kochhar 2009 [30]; Singh and Vallinayagam 2009 [31]; Vallinayagam 2009 [32]; Kumar, N. and Kumar, N. 2020 [33]).

MIS is one of the most important alkaline anorogenic magmatisms in the Indian subcontinent which represents the Pan-African thermos-tectonic episode. The rocks of MIS are granite (hypersolvus and subsolvus), rhyolite, dacite, trachyte, andesite, pyroclasts, basalt, gabbro and dolerite. They have been encountered in the form of ring structures, ring dykes, residual hills, inselbergs and scattered hummocks where the exposures are covered by sand dunes. MIS representatives are well exposed in the Bhiwani district (at Tusham area) of Haryana, Jhunjhunu, Jodhpur, Pali, Barmer (at Siwana, Kundal and Nakora areas), Jalor, Jaisalmer districts of Rajasthan. The representatives of MIS rocks are also reported from Nagar Parkar, Kirana hills in Pakistan (Qasem Jan et al. 1997) [34] (Figure 1).

The term "Malani beds" for a series of volcanics rocks was introduced by Blanford (1877) [35]. La Touche (1902) [36] provided initial accounts on the geology of the suites of rocks belonging to MIS. He first sighted Malani Igneous Suite in which the rocks are well exposed in the erstwhile region of Malani in Western Rajasthan. Many geoscientists have worked to know the petrological, geochemical and petrogenetic aspects of the MIS (La Touche 1902 [36]; Pascoe 1959 [37]; Mukherjee 1962 [38]; Pareek 1981 [2]; Bhushan 1985 [4]; Kochhar et al. 1991 [39]; Dhar et al. 1996 [14]; Vallinayagam and Kochhar 1998 [40]; Bhushan and Chittora 1999 [16]; Kochhar 2000 [41]; Vallinayagam 2001 [21]; Bhushan and Chandrasekaran 2002 [22]; Singh and Vallinayagam 2006 [27]; Sharma, R., and Kumar, N. 2017 [42]; Kumar, N., Kumar, N. and Singh, A. K. 2019 [43]), but the details are still insufficient. So, in the light of the above the present research paper discusses the geochemical observations of the Neoproterozoic volcanic rocks of the Nakora area which is further used to understand the petrogenetic 


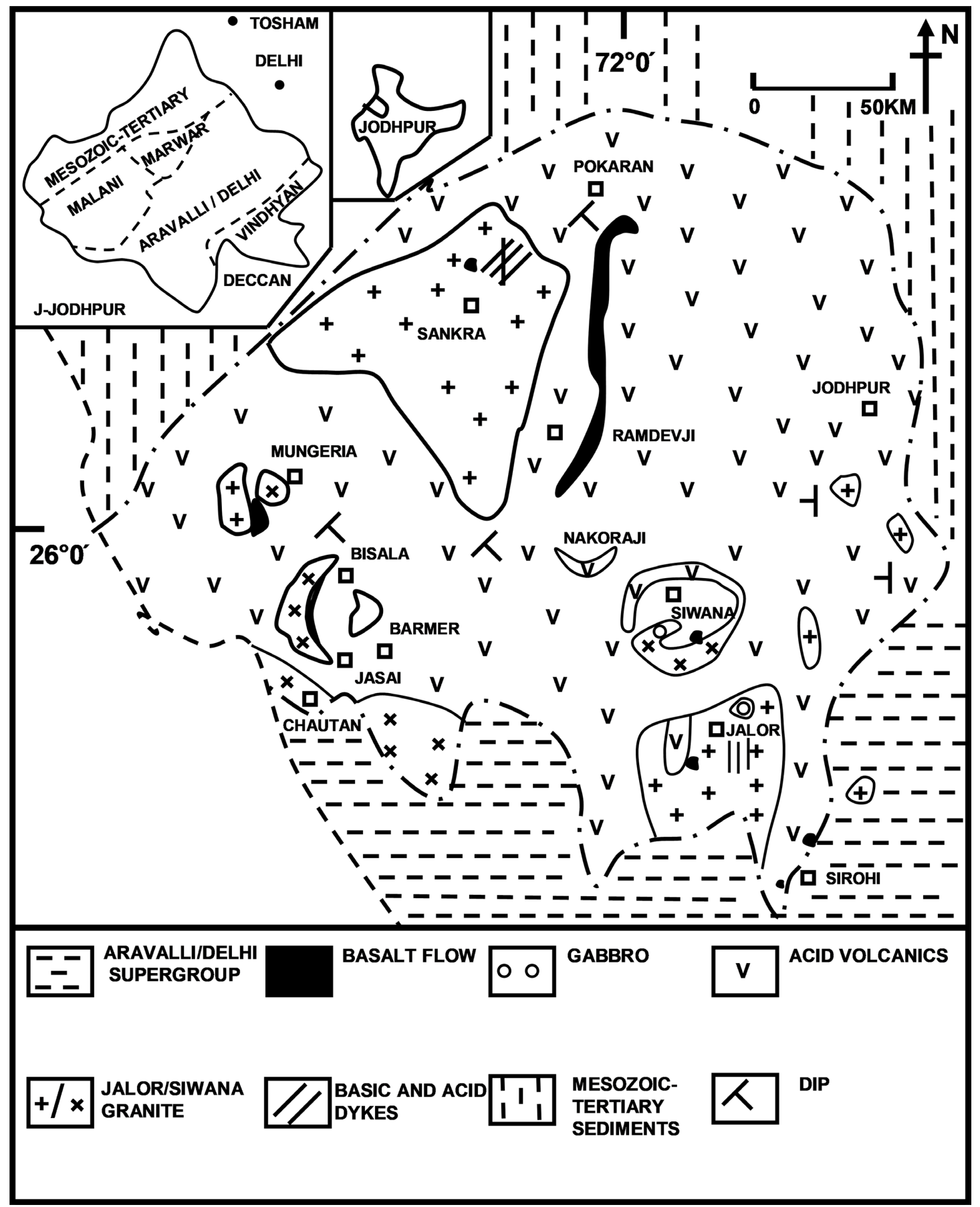

Figure 1. Geological map of Malani Igneous Suite, Rajasthan, India (modified after Vallinayagam 2003) [44].

modelling of the Malani Igneous Suite (MIS).

\section{Geological Setting}

A-type MIS magmatism (55,000 sq. $\mathrm{km})$ is the result of tectono-magmatic event in Western Rajasthan which was related to hot spot activity (Kochhar 1984 [45]; Eby and Kochhar 1990 [8]; Kochhar 2000 [41]). In comparison to intermediate and basic rocks, the NRC has a prevalence of acid volcanic rocks and their oc- 
currence is given below in terms of the extrusive, intrusive and dyke phases.

1) Extrusive phase: Basalt, trachyte, rhyolite, pyroclastic assemblages (ash, tuff, breccia, agglomerate and perlite)

2) Intrusive phase: Gabbro, granite

3) Dyke phase: Basalt, dolerite, rhyolite, microgranite

The above explained rock types occur on the various hills such as Sewadiya hill, Maini hill, Milara hill, Pabre hill, Mewanagar hill, Dadawari hill, Variya and Tikhi hill in the study area (Figure 2). Basalt is fine-grained, with colors ranging from black to dark brown to light greyish brown to dark greyish brown. Basalt shows large size vesicles (approx. $4-6 \mathrm{~mm}$ ) and sometimes these vesicles are permeated by calcite. Basalt xenoliths are observed in the rhyolite (Figure 3(a)). Rhyolite with pyroclastic assemblages may be found in nearly every hill in the NRC, and it is mostly dark brown in colour with various hues of light brown, brick red, grey, green, yellow, blue and purple. It shows both porphyritic and

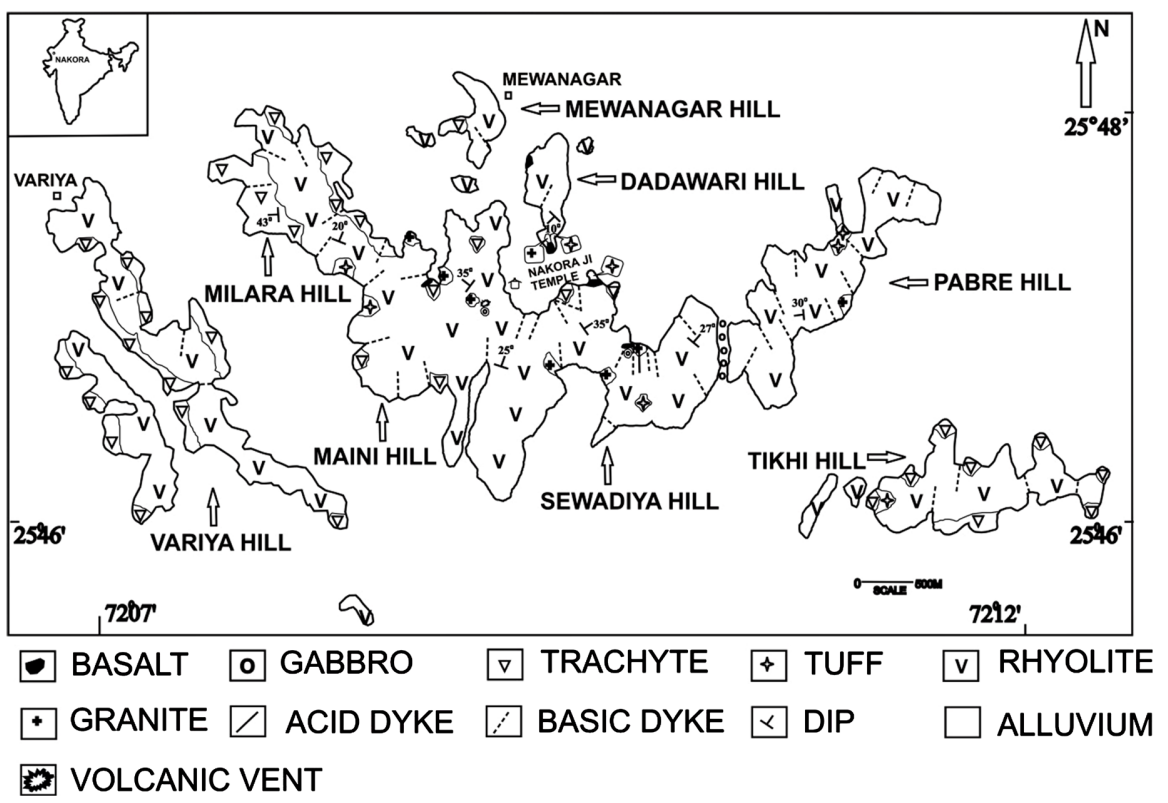

Figure 2. Geological map of Nakora area, Western Rajasthan, India.

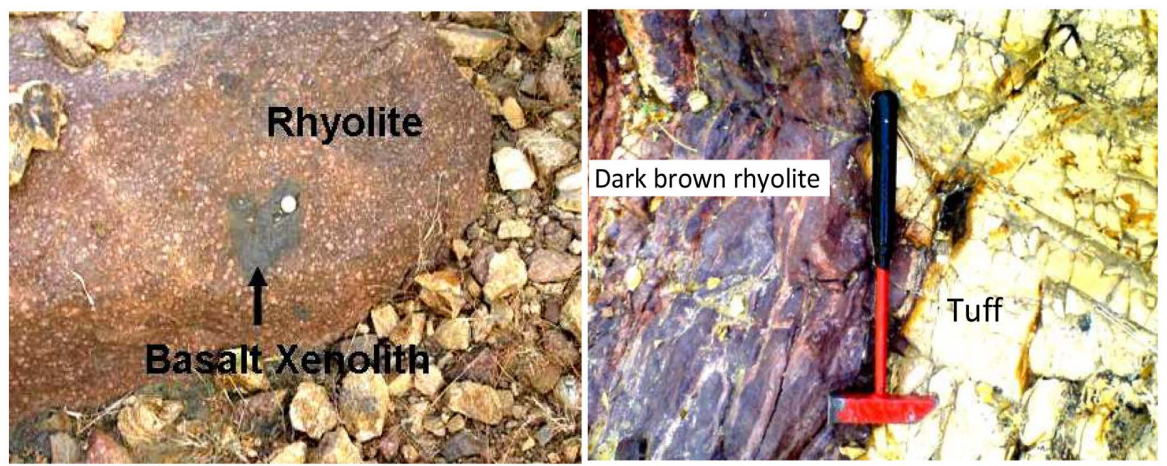

(a)

(b)

Figure 3. (a) Basalt xenoliths observed in the rhyolite; (b) The sharp contact between rhyolite and tuff. 
non-porphyritic nature. Maximum flow thickness in Nakora area i.e. $200 \mathrm{~m}$ represented by dark brown rhyolite at Sewadiya and Maini hill. The sharp contact between rhyolite and tuff (Figure 3(b)) is observed.

\section{Sampling and Analytical Methodology}

Geological field work was carried out in Nakora and adjoining areas for geological mapping (in 1:25,000 scale) using Survey of India topographical sheet no.45C/1 and Brunton Compass. Various rock types exposed in the area were identified and their interrelationships have been delineated based on a thorough field investigation (including the nature of contact between distinct lithological units, the mode of occurrence of rock types, the structure and mineralogy of outcrop rocks, and hand specimens, study of flows, intrusions, xenoliths, dykes, veins, alterations etc.). Flow stratigraphy of the area is established and various volcanic features were studied. Important rock samples were collected (in chips $3 \times 5 \times 7 \mathrm{~cm}$ and in blocks $6 \times 10 \times 10 \mathrm{~cm}$ ) using geological hammer and chisels for petrographical and geochemical investigations. Field photography has been carried out depicting the important characters and interrelationships among various lithological units exposed in the study area. Petromineralogical investigations (including modal analysis and photomicrography) were carried out by using Leitz 12 pol S microscope with Swift Counter and Photoautomat.

Based on the field and petromineralogical studies, 39 samples were selected and analyzed for major elements $\left(\mathrm{SiO}_{2}, \mathrm{TiO}_{2}, \mathrm{Al}_{2} \mathrm{O}_{3}, \mathrm{Fe}_{2} \mathrm{O}_{3}, \mathrm{MnO}, \mathrm{MgO}, \mathrm{CaO}\right.$ and $\mathrm{P}_{2} \mathrm{O}_{5}$ ) by using wet chemical method after Shapiro and Brannock (1962) [46] using a Systronics UV-VIS spectrophotometer-108 and Atomic Absorption Spectrophotometer (Varian 240 FS-AAS) at Department of Geology, Kurukshetra University, Kurukshetra; alkalies $\left(\mathrm{Na}_{2} \mathrm{O}\right.$ and $\left.\mathrm{K}_{2} \mathrm{O}\right)$ were determined using Systronics Mediflame Photometer-128 at Department of Chemistry, Kurukshetra University, Kurukshetra. 25 samples (9 granites, 6 rhyolites, 1 trachyte, 1 tuff, 5 basalts, 2 gabbros, 1 dolerite) were analyzed for trace (Rb, Ba, Sr, Zr, Y, V, Cr, $\mathrm{Co}, \mathrm{Ni}, \mathrm{Zn}, \mathrm{Ga}, \mathrm{Sc}, \mathrm{U}, \mathrm{Th}, \mathrm{Pb}$ and $\mathrm{Nb}$ ) and rare earth elements (La, Ce, Pr, Nd, $\mathrm{Sm}, \mathrm{Eu}, \mathrm{Gd}, \mathrm{Tb}, \mathrm{Dy}, \mathrm{Ho}, \mathrm{Er}, \mathrm{Tm}, \mathrm{Yb}$ and Lu) by ICP-MS (PerkinElmer Sciex ELAN DRC II) at National Geophysical Research Institute (NGRI), Hyderabad. Standardization for major and trace elements including rare earth elements was based on USGS rock standards RGM-1, JG-2, MRG-1. The analytical precision is found to be in the error level of $<5 \%$ for major and $<10 \%$ for trace elements.

\section{Geochemistry: Major Elements}

Geochemical data is used to determine the rock nomenclature/classifications, to depict chemical variations, the nature of the igneous suite and the tectonic environment and post magmatic process, pressure and temperature conditions in the development of the suite of rocks. The norms suggest the over and undersaturation of silica as the presence of quartz in the norm shows that the rocks are oversaturated with silica; the presence of olivine, perovskite, nepheline, leucite, 
calcium, and orthosilicate in the norm indicates that the rocks are undersaturated with silica. The presence of corundum, on the other hand, suggests that the rocks contain an excessive amount of alumina. The excessive amount of iron is indicated by the presence of hematite and magnetite. After the formation of anorthite, diopside is formed when there is an excess of $\mathrm{CaO}$. After the development of feldspars, the presence of an excessive amount of $\mathrm{CaO}$ allows wollastonite and calcite to form. The presence of acmite, soda, and potash metasilicates in the rocks supports their alkaline character.

Basalt: In basalts samples, $\mathrm{SiO}_{2}, \mathrm{Al}_{2} \mathrm{O}_{3}$ and total alkalies $\left(\mathrm{Na}_{2} \mathrm{O}, \mathrm{K}_{2} \mathrm{O}\right.$ ) (in wt\%) ranges from 45.59 - 54.00, 11.20 - 17.40 and 2.85 - 7.17 (1.00 - 2.71, 1.85 - 4.46) respectively. Total iron $\left(\mathrm{Fe}_{2} \mathrm{O}_{3}\right), \mathrm{TiO}_{2}, \mathrm{CaO}, \mathrm{MgO}, \mathrm{MnO}$ and $\mathrm{P}_{2} \mathrm{O}_{5}$ (in wt\%) ranges from 9.67 - 14.50, 2.34 - 4.12, 1.68 - 7.60, 4.42 - 6.56, 1.62 - 2.70 and 0.31 0.88 respectively (Table 1 ).

Trachyte: In trachyte, $\mathrm{SiO}_{2}$ varies from $66.7 \mathrm{wt} \%$ to $69.4 \mathrm{wt} \%$ (Table 1). $\mathrm{Al}_{2} \mathrm{O}_{3}$ ranges from $6.1 \mathrm{wt} \%$ to $10.5 \mathrm{wt} \%$. Total alkalies $\left(\mathrm{Na}_{2} \mathrm{O}, \mathrm{K}_{2} \mathrm{O}\right)$ and total iron $\left(\mathrm{Fe}_{2} \mathrm{O}_{3}\right)$ show $4.36-8.37(1.06-1.09,4.3-7.28)$ and $8.1 \mathrm{wt} \%-10.2 \mathrm{wt} \%$ respectively. $\mathrm{TiO}_{2}, \mathrm{CaO}, \mathrm{MgO}, \mathrm{MnO}$ and $\mathrm{P}_{2} \mathrm{O}_{5}$ (in wt\%) ranges from 2.67 - 2.93, 0.8 $1.52,0.49-1.47,1.25-3.13$ and $0.5-0.55$ respectively.

Rhyolite: As compared to trachytes, the rhyolites show (wt\%) high $\mathrm{SiO}_{2}$ (64.80 - 71.90), high $\mathrm{Al}_{2} \mathrm{O}_{3}\left(7.20\right.$ - 13.10), low total alkalies (5.42 - 7.99) $\left(\mathrm{Na}_{2} \mathrm{O}\right.$ : $0.94-1.18, \mathrm{~K}_{2} \mathrm{O}: 4.48$ - 6.81), low total iron (5.40 - 9.70), low $\mathrm{TiO}_{2}(1.61-2.84)$, high $\mathrm{CaO}(0.80$ - 3.64) and high $\mathrm{MgO}(0.49$ - 3.28).

In normative composition, quartz ranges from 29.20 wt\%to $46.37 \mathrm{wt} \%$. Orthoclase varies from 26.47 to 40.24 whereas DI ranges from 72.76 to 84.67 . AI content varies from 0.55 to 1.19 and average value is 0.84 . AI value is less than 1 for ten samples (sample no. 27, 28, 118, 124, 126, 135, 152, 35, 113 and 82) which indicate peraluminous nature of rhyolites except six samples (no. 104, 164, 170, 15, 48 and 116) indicating the peralkaline nature (Giret et al. 1980) [47]. Alkali nature of rhyolites is also indicated by presence of acmite in above samples (no. 104, 164, 170, 15, 48 and 116). The samples (no. 28, 118, 124, 126, 135, 152, 35, 113 and 82 ) which show corundum (ranges from 0.29 to 5.38 ) indicate the aluminous nature of the rocks.

Tuff: As compare to rhyolites, the tuff shows low $\mathrm{SiO}_{2}$ which varies from $64.90 \mathrm{wt} \%$ to $66.60 \mathrm{wt} \%$ (Table 1 ). $\mathrm{Al}_{2} \mathrm{O}_{3}$ ranges from 10.2 to $13.8 \mathrm{wt} \%$. Low total alkalies $\left(\mathrm{Na}_{2} \mathrm{O}, \mathrm{K}_{2} \mathrm{O}\right)$ and low total iron $\left(\mathrm{Fe}_{2} \mathrm{O}_{3}\right)$ varies from 5.87 - $6.92(0.94$ $1.10,4.93$ - 5.82) and $7.2 \mathrm{wt} \%-8.9 \mathrm{wt} \%$ respectively. High $\mathrm{TiO}_{2}$, low $\mathrm{CaO}$, low $\mathrm{MgO}, \mathrm{MnO}$ and $\mathrm{P}_{2} \mathrm{O}_{5}$ (in wt\%) ranges from $2.6-3.16,0.56-1.96,2.13-2.56$, $0.75-1.25$ and $0.58-0.65$ respectively.

Total Alkali-Silica (TAS) diagram: The Nakora acid volcanics and basic rocks (basalts) are plotted in the TAS diagram (Figure 4) (Le Bas et al. 1986) [48]. The Nakora rhyolites lie in the field of rhyolite and dacite however dacite is very close to rhyolite. The basaltic rocks lie in the field of basalt, trachybasalt, basaltic trachyandesite and basaltic andesite. The trachytes of Nakora area lie in the field of trachydacite. 
Table 1. Major element data (in wt\%) of Nakora rocks, Barmer District, Rajasthan.

\begin{tabular}{|c|c|c|c|c|c|c|c|c|}
\hline \multirow[b]{2}{*}{$\begin{array}{c}\text { ROCK TYPE } \\
\text { OXIDES }\end{array}$} & \multicolumn{2}{|c|}{ RHYOLITE } & \multicolumn{2}{|c|}{ TRACHYTE } & \multicolumn{2}{|c|}{ TUFF } & \multicolumn{2}{|c|}{ BASALT } \\
\hline & $\begin{array}{l}\text { Maximum } \\
\text { value (with } \\
\text { sample } \\
\text { number) }\end{array}$ & $\begin{array}{l}\text { Minimum } \\
\text { value (with } \\
\text { sample } \\
\text { number) }\end{array}$ & $\begin{array}{l}\text { Maximum } \\
\text { value } \\
\text { (with } \\
\text { sample } \\
\text { number) }\end{array}$ & $\begin{array}{l}\text { Minimum } \\
\text { value (with } \\
\text { sample } \\
\text { number) }\end{array}$ & $\begin{array}{l}\text { Maximum } \\
\text { value } \\
\text { (with } \\
\text { sample } \\
\text { number) }\end{array}$ & $\begin{array}{l}\text { Minimum } \\
\text { value (with } \\
\text { sample } \\
\text { number) }\end{array}$ & $\begin{array}{c}\text { Maximum } \\
\text { value (with } \\
\text { sample } \\
\text { number) }\end{array}$ & $\begin{array}{c}\text { Minimum } \\
\text { value (with } \\
\text { sample } \\
\text { number) }\end{array}$ \\
\hline $\mathrm{SiO}_{2}$ & $71.9(35)$ & $64.8(113)$ & $69.4(51)$ & $66.7(63)$ & $66.6(13)$ & $64.9(89)$ & $54(154)$ & $45.59(159\{2\})$ \\
\hline $\mathrm{TiO}_{2}$ & $2.84(116)$ & $1.61(152)$ & $2.93(51)$ & $2.67(63)$ & $3.16(13)$ & $2.6(89)$ & $4.12(6)$ & $2.34(5)$ \\
\hline $\mathrm{Al}_{2} \mathrm{O}_{3}$ & $13.1(28)$ & $7.2(116)$ & $10.5(56)$ & $6.1(63)$ & $13.8(2)$ & $10.2(13)$ & $17.4(159\{2\})$ & $11.2(160 a)$ \\
\hline $\mathrm{Fe}_{2} \mathrm{O}_{3}$ & $9.7(35)$ & $5.4(48)$ & $10.2(63)$ & $8.1(56)$ & $8.9(13)$ & $7.2(2)$ & $14.5(159\{2\})$ & $9.67(160 a)$ \\
\hline $\mathrm{MnO}$ & $1.9(170)$ & $0.13(152)$ & $3.13(56)$ & $1.25(51)$ & $1.25(13)$ & $0.75(89)$ & $2.7(160 a)$ & $1.62(159\{2\})$ \\
\hline $\mathrm{MgO}$ & $3.28(135)$ & $0.49(104)$ & $1.47(56)$ & $0.49(51)$ & $2.56(13)$ & $2.13(89)$ & $6.56(160 a)$ & $4.42(154)$ \\
\hline $\mathrm{CaO}$ & $3.64(164)$ & $0.8(135)$ & $1.52(51)$ & $0.8(56)$ & $1.96(89)$ & $0.56(13)$ & $7.6(154)$ & $1.68(6)$ \\
\hline $\mathrm{Na}_{2} \mathrm{O}$ & $1.18(164,170)$ & $0.94(116)$ & $1.09(63)$ & $1.06(56)$ & $1.1(89)$ & $0.94(13)$ & $2.71(160 a)$ & $1(6)$ \\
\hline $\mathrm{K}_{2} \mathrm{O}$ & $6.71(15)$ & $4.48(35)$ & $7.28(63)$ & $4.3(56)$ & $5.82(89)$ & $4.93(2)$ & $4.46(5)$ & $1.85(154)$ \\
\hline $\mathrm{P}_{2} \mathrm{O}_{5}$ & $0.66(113)$ & $0.32(104)$ & $0.55(56)$ & $0.5(63)$ & $0.65(89)$ & $0.58(13)$ & $0.88(160 a)$ & $0.31(159\{2\})$ \\
\hline $\mathrm{H}_{2} \mathrm{O}$ & $0.5(15)$ & $0.1(116,126)$ & $0.2(63)$ & $0.1(51,56)$ & $0.4(13)$ & $0.2(2,89)$ & $0.8(159\{2\})$ & $0.3(154,6)$ \\
\hline \multicolumn{9}{|l|}{ CIPW Norms } \\
\hline Quartz & $46.4(35)$ & $29.2(28)$ & $42.72(56)$ & $37.4(63)$ & $37.25(2)$ & $30.69(89)$ & $23.27(6)$ & $3.71(159\{2\})$ \\
\hline Orthoclase & $39.7(15)$ & $26.5(35)$ & $37.29(51)$ & $25.41(56)$ & $34.39(89)$ & $29.13(2)$ & $26.36(5)$ & $10.93(154)$ \\
\hline Albite & $9.22(135,152)$ & $2.85(170)$ & $8.97(56)$ & $1.9(51)$ & $9.31(89)$ & $7.95(13)$ & $22.93(160 a)$ & $8.46(6)$ \\
\hline Anorthite & $8.25(28)$ & $0.44(135)$ & $0.38(56)$ & --- & $5.48(89)$ & $0.75(2)$ & $30.22(159\{2\})$ & $5.79(6)$ \\
\hline Corundm & $5.38(82)$ & $0.29(35)$ & $3.96(56)$ & --- & $3.11(13)$ & --- & --- & --- \\
\hline Diopside & $8.08(170)$ & $2.63(104)$ & $0.65(63)$ & --- & $7.05(2)$ & $2.18(89)$ & $7.64(6)$ & $0.79(159\{2\})$ \\
\hline Hypersthene & $8.17(135)$ & $0.34(170)$ & $3.66(56)$ & $1.22(51)$ & $6.38(13)$ & --- & $9.7(160 a)$ & $2.72(154)$ \\
\hline Wollastonite & $0.72(164)$ & $0.21(104)$ & $2.69(63)$ & --- & $6.13(2)$ & $5.3(89)$ & $15.44(159\{2\})$ & $9.75(154)$ \\
\hline Olivine & --- & --- & $8.13(63)$ & --- & --- & --- & --- & --- \\
\hline Acmite & $6.29(170)$ & $0.84(48)$ & $6.3(51)$ & $1.78(56)$ & --- & --- & --- & --- \\
\hline Illmenite & $6.28(152)$ & $0.41(15,82)$ & $5.53(56)$ & $2.67(51)$ & $2.67(13)$ & $1.6(89)$ & $5.78(160 a)$ & $3.47(159\{2\})$ \\
\hline Hematite & $9.7(35)$ & $5.11(48)$ & $7.39(63)$ & $6.52(51)$ & $8.9(13)$ & $7.2(2)$ & $14.5(159\{2\})$ & $9.67(160 a)$ \\
\hline Apatite & $1.53(113)$ & $0.74(104)$ & $1.27(56)$ & $1.16(63)$ & $1.51(89)$ & $1.34(13)$ & $2.04(160 a)$ & $0.72(159\{2\})$ \\
\hline Sphene & $5.14(15)$ & $0.49(170)$ & $2.83(51)$ & $1.02(63)$ & $2.59(2)$ & $2.24(89)$ & $1.92(6)$ & $0.79(159\{2\})$ \\
\hline Rutile & $2.48(82)$ & $0.81(48)$ & $0.47(51)$ & --- & $2.24(13)$ & --- & $3.81(154)$ & $1.04(5)$ \\
\hline DI & $84.7(104)$ & $72.76(164)$ & $78.26(51)$ & $70.71(63)$ & $77.08(13)$ & 74.39 (89) & $54.07(6)$ & $33.37(159\{2\})$ \\
\hline AI & $1.19(170)$ & $0.55(82)$ & $1.59(63)$ & $0.62(56)$ & $0.73(13)$ & $0.51(2)$ & $0.73(160 a)$ & $0.31(159\{2\})$ \\
\hline $\mathrm{Na}+\mathrm{K}$ & $7.92(104)$ & $5.52(35)$ & $8.37(63)$ & $5.36(56)$ & $6.92(89)$ & $6(2)$ & $6.5(160 a)$ & $3.04(154)$ \\
\hline $\mathrm{Na} / \mathrm{K}$ & $0.24(135)$ & $0.15(28)$ & $0.25(56)$ & $0.15(63)$ & $0.22(2)$ & $0.17(13,89)$ & $0.72(160 a)$ & $0.23(5)$ \\
\hline $\mathrm{KN} / \mathrm{C}$ & $7.06(135)$ & $2.19(15)$ & $7.47(63)$ & $4.85(51)$ & $8(13)$ & $1.66(2)$ & $2.85(6)$ & $0.4(154)$ \\
\hline $\mathrm{A} / \mathrm{CNK}$ & $1.44(82)$ & $0.47(164)$ & $1.43(56)$ & $0.55(63)$ & $1.75(2)$ & $1(89)$ & $1.67(6)$ & $0.12(160 a)$ \\
\hline$A n / A n+b$ & $0.51(28)$ & $0.05(135)$ & $0.04(56)$ & --- & $0.37(89)$ & $0.08(2)$ & $0.76(159\{2\})$ & $0.24(160 a)$ \\
\hline $\mathrm{Mg \#}$ & --- & --- & --- & --- & --- & --- & $54.1(154)$ & $45.9(159\{2\})$ \\
\hline
\end{tabular}




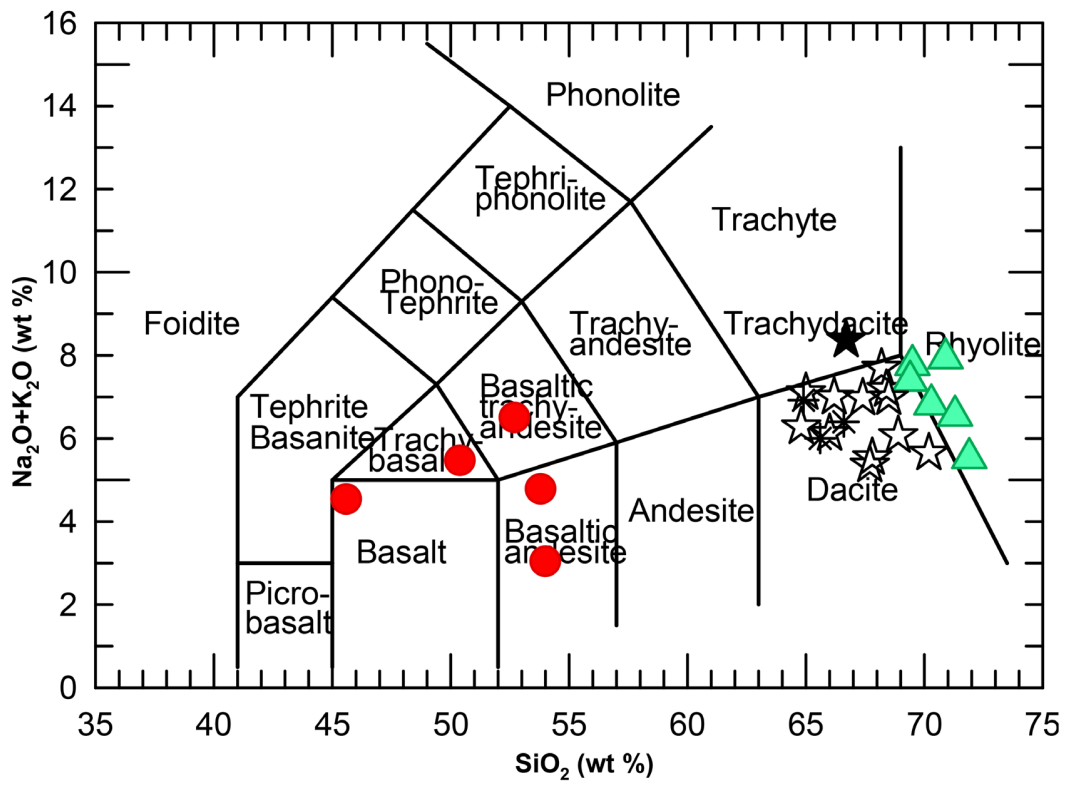

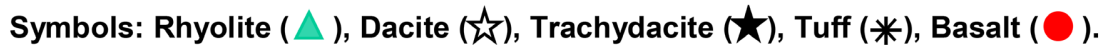

Figure 4. Total Alkali Silica (TAS) diagram showing the classification of Nakora Volcanics.

\section{Geochemistry: Trace and Rare Earth Elements}

Basalt: In basalts, $\mathrm{Ni}, \mathrm{Cu}$ and $\mathrm{Zn}$ ranges from 10.69 - 69.28, 23.08 - 50.14 and 127.63 - 293.47 (in ppm) respectively (Table 2). Average values of $\mathrm{Ni}, \mathrm{Cu}$ and $\mathrm{Zn}$ are 35.05, 35.18 and 190.09 respectively. $\mathrm{Rb}, \mathrm{Sr}, \mathrm{Y}$ and $\mathrm{Zr}$ show a range (average) from 41.42 - 87.13 (60.47), 140.88 - 214.83 (178.34), 42.9 - 156.22 (74.87) and 97.81 - 1604.39 (501.98) respectively. The radioactive elements (Th and U) ranges from 1.28 - 6.88 (average 2.92) and 0.17 - 1.04 (average 0.41 ) respectively. In the rare earth elements (Table 3), $\mathrm{La}, \mathrm{Ce}, \mathrm{Eu}$ and $\mathrm{Yb}$ ranges from 15.55 $90.77,48.55-211.55,3.19-6.64$ and $2.34-11.19$ and average values are 34.91 , $86.74,4.05$ and 5.43 respectively. $\mathrm{Eu} / \mathrm{Eu}^{*}$ varies from $0.24-0.38$ with average of 0.31 .

Trachyte: It has $\mathrm{Ni}$ (23.09), $\mathrm{Cu}$ (9.88), $\mathrm{Zn}$ (226.61), Rb (144.69), $\mathrm{Sr}$ (11.94), Y (227.51), Zr (2009.4), Th (17.39) and U (1.78) concentrations in ppm. In REE, $\mathrm{La}, \mathrm{Ce}, \mathrm{Nd}, \mathrm{Sm}, \mathrm{Eu}$ and $\mathrm{Yb}$ show 94.05, 243.67, 133.58, 30.08, 2.24 and $17 \mathrm{ppm}$ concentration with $\mathrm{Eu} / \mathrm{Eu}^{*}$ ratio of $0.08 \mathrm{ppm}$.

Rhyolite: As compared to trachyte, it shows low ranges of $\mathrm{Ni}, \mathrm{Cu}, \mathrm{Zn}$ and $\mathrm{Rb}$ and vary from $1.64-3.27,0.22-0.54,20.79-57.58$ and $79.03-138.43$ respectively. Sr, Y, Zr, Th and U show high values and ranges from 8.5 - 16.8, 99.44 240.18, 786.43 - 4053.14, 7.18 - 19.71 and 1.36 - 3.07 respectively. In REE, La, Ce, $\mathrm{Nd}, \mathrm{Sm}, \mathrm{Eu}$ and $\mathrm{Yb}$ ranges from 13.09 - 91.68 (low), 45.98 - 188.27 (low), 46.56 137.53 (high), 10.55 - 31.84 (high), 0.76 - 2.51 (high) and 10.98 - 25.89 (high).

Tuff: As compared to average concentrations in rhyolite, it shows low $\mathrm{Ni}$ (1.68), low $\mathrm{Cu}$ (0.18), low $\mathrm{Zn}$ (30.06), high Rb (184.02), low $\mathrm{Sr}$ (12.31), high $\mathrm{Y}$ (306.05), high $\mathrm{Zr}$ (2500.05), high Th (22.57) and high U (5.92) concentrations in ppm. In REE, La, Ce, Nd, Sm, Eu and Yb show low 35.38, low 212.07, high 
Table 2. Trace element data (in ppm) of Nakora rocks, Barmer District, Rajasthan.

\begin{tabular}{|c|c|c|c|c|c|c|}
\hline \multirow[b]{2}{*}{ ROCK TYPE } & \multicolumn{2}{|c|}{ RHYOLITE } & \multirow{2}{*}{$\begin{array}{c}\text { TRACHYTE } \\
\text { Value from } \\
\text { single sample } \\
\text { (59) }\end{array}$} & \multirow{2}{*}{$\begin{array}{c}\text { TUFF } \\
\text { Value from } \\
\text { single } \\
\text { sample (89) }\end{array}$} & \multicolumn{2}{|c|}{ BASALT } \\
\hline & $\begin{array}{l}\text { Maximum value } \\
\text { (with sample } \\
\text { number) }\end{array}$ & $\begin{array}{l}\text { Minimum value } \\
\text { (with sample } \\
\text { number) }\end{array}$ & & & $\begin{array}{l}\text { Maximum value } \\
\text { (with sample } \\
\text { number) }\end{array}$ & $\begin{array}{c}\text { Minimum value } \\
\text { (with sample } \\
\text { number) }\end{array}$ \\
\hline Sc & $2.29(126)$ & $1.13(15)$ & 2 & 1.82 & $35.52(154)$ & $26.25(6)$ \\
\hline $\mathrm{V}$ & $4.99(28)$ & $1.61(15)$ & 4.15 & 3.68 & $247.32(154)$ & $106.42(5)$ \\
\hline $\mathrm{Cr}$ & $16.89(126)$ & $1.53(28)$ & 23.68 & 4.62 & $67.48\{159(2)\}$ & $23.52(154)$ \\
\hline Co & $0.87(126)$ & $0.38(27)$ & 0.61 & 0.62 & $36.47(6)$ & $18.85(5)$ \\
\hline $\mathrm{Ni}$ & $3.27(164)$ & $1.64(28)$ & 23.09 & 1.68 & $69.28\{159(2)\}$ & $10.69(154)$ \\
\hline $\mathrm{Cu}$ & $0.54(27)$ & $0.22(164,15)$ & 9.88 & 0.18 & $50.14(160 a)$ & $23.08(154)$ \\
\hline $\mathrm{Zn}$ & $57.58(126)$ & $20.79(28)$ & 226.61 & 30.06 & $293.47(154)$ & $127.63(160 a)$ \\
\hline $\mathrm{Ga}$ & $33.54(126)$ & $23.71(28)$ & 30.76 & 27.44 & $42.09(5)$ & $18.61(6)$ \\
\hline $\mathrm{Rb}$ & $138.43(126)$ & $79.03(164)$ & 144.69 & 184.02 & $87.13(5)$ & $41.42(154)$ \\
\hline $\mathrm{Sr}$ & $16.8(28)$ & $8.5(15)$ & 11.94 & 12.31 & $214.83(6)$ & $140.88(5)$ \\
\hline $\mathrm{Y}$ & $240.18(28)$ & $99.44(15)$ & 227.51 & 306.05 & $156.22(5)$ & $42.9(6)$ \\
\hline $\mathrm{Zr}$ & $4053.14(28)$ & 786.43 (124) & 2009.4 & 2500.05 & $1604.39(5)$ & $97.81(160 a)$ \\
\hline $\mathrm{Nb}$ & $81.24(28)$ & $20.97(164)$ & 82.57 & 52 & $78.96(5)$ & $6.82(154)$ \\
\hline Cs & $1(27)$ & $0.31(15)$ & 0.74 & 0.98 & $1.34(5)$ & $0.82(160 a)$ \\
\hline $\mathrm{Ba}$ & $132.48(27)$ & $52.15(154)$ & 80.29 & 100 & $407.11(154)$ & $286.58\{159(\mathrm{a})\}$ \\
\hline $\mathrm{Hf}$ & $54.38(28)$ & $24.01(124)$ & 50.44 & 75.54 & $33.17(5)$ & $2.43(160 a)$ \\
\hline $\mathrm{Ta}$ & $2.42(126)$ & $1.23(27)$ & 8.44 & 2.23 & $7.03(5)$ & $0.36(160 a)$ \\
\hline $\mathrm{Pb}$ & $25.13(27)$ & $3.84(15)$ & 2.92 & 5.82 & $4.85(154)$ & $1.29(6)$ \\
\hline Th & $19.71(28)$ & $7.18(164)$ & 17.39 & 22.57 & $6.88(5)$ & $1.28(6)$ \\
\hline $\mathrm{U}$ & $3.58(126)$ & $1.36(15)$ & 1.78 & 5.92 & $1.04(5)$ & $0.17(160 a, 154)$ \\
\hline $\mathrm{Ba} / \mathrm{Rb}$ & $1.56(164)$ & $0.6(124)$ & 0.55 & 0.54 & $9.83(154)$ & $3.56\{159(2)\}$ \\
\hline $\mathrm{Rb} / \mathrm{Sr}$ & $11.12(15)$ & $5.61(28)$ & 12.12 & 14.95 & $0.62(5)$ & $0.22(6)$ \\
\hline $\mathrm{Zr} / \mathrm{Rb}$ & $42.99(28)$ & $7.9(27)$ & 13.89 & 13.59 & $18.41(5)$ & $2.14(160 a)$ \\
\hline $\mathrm{Ba} / \mathrm{Sr}$ & $12.94(164)$ & $4.56(28)$ & 6.72 & 8.12 & $2.56(154)$ & $1.53\{159(2)\}$ \\
\hline $\mathrm{Sr} / \mathrm{Y}$ & $0.1(27)$ & $0.07(28,124)$ & 0.05 & 0.04 & $5.01(6)$ & $0.9(5)$ \\
\hline $\mathrm{Nb} / \mathrm{Y}$ & $0.36(15)$ & $0.18(164,124)$ & 0.36 & 0.17 & $0.51(5)$ & $0.09(154)$ \\
\hline $\mathrm{Zr} / \mathrm{Nb}$ & $49.89(28)$ & $22.67(15)$ & 24.34 & 48.08 & $24.89\{159(2)\}$ & $11.11(160 a)$ \\
\hline $\mathrm{Zr} / \mathrm{Y}$ & $16.88(28)$ & $4.8(124)$ & 8.83 & 8.17 & $10.27(5)$ & $1.37(154)$ \\
\hline $\mathrm{Y} / \mathrm{Nb}$ & $5.65(124)$ & $2.75(15)$ & 2.76 & 5.89 & $11.55(154)$ & $1.98(5)$ \\
\hline $\mathrm{K} / \mathrm{Rb}$ & $0.06(15,164)$ & $0.03(126)$ & 0.02 & 0.03 & $0.07(160 a)$ & $0.03\{159(2)\}$ \\
\hline
\end{tabular}


Table 3. Rare earth element data (in ppm) of Nakora rocks, Barmer District, Rajasthan.

\begin{tabular}{|c|c|c|c|c|c|c|}
\hline \multirow[b]{2}{*}{ ROCK TYPE } & \multicolumn{2}{|c|}{ RHYOLITE } & \multirow{2}{*}{$\begin{array}{c}\text { TRACHYTE } \\
\text { Value from } \\
\text { single sample } \\
(56)\end{array}$} & \multirow{2}{*}{$\begin{array}{c}\text { TUFF } \\
\text { Value from } \\
\text { single sample } \\
\text { (89) }\end{array}$} & \multicolumn{2}{|c|}{ BASALT } \\
\hline & $\begin{array}{c}\text { Maximum value } \\
\text { (with sample } \\
\text { number) }\end{array}$ & $\begin{array}{c}\text { Minimum value } \\
\text { (with sample } \\
\text { number) }\end{array}$ & & & $\begin{array}{l}\text { Maximum value } \\
\text { (with sample } \\
\text { number) }\end{array}$ & $\begin{array}{c}\text { Minimum value } \\
\text { (with sample } \\
\text { number) }\end{array}$ \\
\hline $\mathrm{La}$ & $91.68(28)$ & $13.09(15)$ & 94.05 & 35.38 & $90.77(5)$ & $15.55(154)$ \\
\hline $\mathrm{Ce}$ & $188.27(28)$ & $45.98(15)$ & 243.67 & 212.07 & $211.51(5)$ & $48.55(154)$ \\
\hline $\operatorname{Pr}$ & $29.9(28)$ & $10.5(15)$ & 26.55 & 23.12 & $25.47(5)$ & $6.52(160 a)$ \\
\hline $\mathrm{Nd}$ & $137.53(28)$ & $46.56(15)$ & 133.58 & 102.38 & $129.09(5)$ & $38.66(160 a)$ \\
\hline $\mathrm{Sm}$ & $31.84(28)$ & $10.55(15)$ & 30.08 & 28.03 & $28.22(5)$ & $9.1(160 \mathrm{a})$ \\
\hline $\mathrm{Eu}$ & $2.51(28)$ & $0.76(15)$ & 2.24 & 1.67 & $6.64(5)$ & 3.19 (160a) \\
\hline $\mathrm{Gd}$ & $28.24(28)$ & $8.81(15)$ & 29.27 & 26.89 & $27.1(5)$ & $9.15(6)$ \\
\hline $\mathrm{Tb}$ & $5.54(28)$ & $1.91(15)$ & 4.5 & 6.68 & $3.85(5)$ & $1.2(6)$ \\
\hline Dy & $41.3(28)$ & $16.3(15)$ & 33.37 & 52.36 & $26.37(5)$ & $7.66(6)$ \\
\hline Ho & $4.9(28)$ & $2.15(15)$ & 7.14 & 6.35 & $5.32(5)$ & $1.42(6)$ \\
\hline Er & $17.48(28)$ & $7.68(15)$ & 20.64 & 22 & $14.31(5)$ & $3.57(6)$ \\
\hline $\mathrm{Tm}$ & $2.43(28)$ & $1.06(15)$ & 2.63 & 3.01 & $1.72(5)$ & $0.37(6)$ \\
\hline $\mathrm{Yb}$ & $25.89(28)$ & $10.98(15)$ & 17 & 31.08 & $11.19(5)$ & $2.34(6)$ \\
\hline $\mathrm{Lu}$ & $4.31(28)$ & $1.73(15)$ & 3.69 & 5.01 & $2.6(5)$ & $0.52(6)$ \\
\hline $\mathrm{Ce} / \mathrm{Nd}$ & $1.59(126)$ & $0.99(15)$ & 1.82 & 2.07 & $1.64(5)$ & $1.13(154)$ \\
\hline $\mathrm{Ce} / \mathrm{Sm}$ & 7.07 (126) & $4.36(15)$ & 8.1 & 7.57 & $7.5(5)$ & $4.18(154)$ \\
\hline $\mathrm{Gd} / \mathrm{Yb}$ & $1.41(124)$ & $0.8(15)$ & 1.72 & 0.87 & $3.91(6)$ & $2.18(154)$ \\
\hline $\mathrm{Ce} / \mathrm{Yb}$ & $11.38(126)$ & $4.19(15)$ & 14.33 & 6.82 & $23.07(6)$ & $7.3(154)$ \\
\hline $\mathrm{Eu} / \mathrm{Eu}^{*}$ & $0.12(27)$ & $0.06(164)$ & 0.08 & 0.06 & $0.38(6)$ & $0.24(5)$ \\
\hline
\end{tabular}

102.38, high 28.03, high 1.67 and high 31.08 ppm concentrations respectively. $\mathrm{Eu} / \mathrm{Eu}^{*}$ ratio shows $0.06 \mathrm{ppm}$ concentration.

$\mathrm{Nb} / \mathrm{Y}$ vs $\mathrm{Zr} / \mathrm{TiO}_{2}$ diagram (Winchester and Floyd 1977) [49] is used in which the selected samples of Nakora volcanics are plotted. The selected acid volcanic rocks are lying in the field of rhyodacite/dacite and the Nakora basic rocks lie in the field of andesite/basalt (Figure 5).

\section{Primitive Mantle Normalized Patterns of Trace Elements}

The Nakora acid volcanic rocks have high LREE enrichment than the HREE which is observed in primitive mantle normalized multi-element diagram using normalization values from Sun and McDonough (1989) [50] and show quite parallel pattern (Figure 6). All the samples show negative $\mathrm{Ba}, \mathrm{Sr}, \mathrm{Eu}$ and $\mathrm{Ti}$ anomalies. Sr and Eu depletion is thought to be linked to feldspar fractionation or their retention in refractory minerals resistant to melting in the lower 


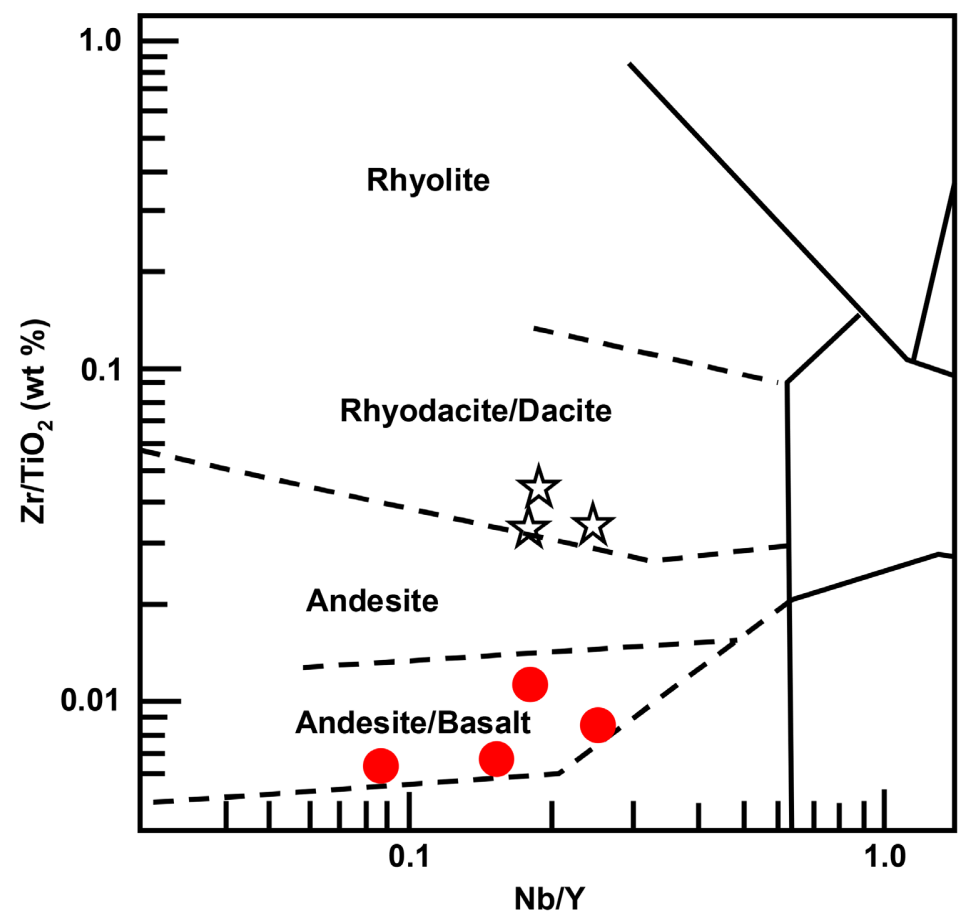

Symbols: Rhyolite ( $\Delta$ ), Dacite (负), Trachydacite ( $九$ ), Tuff(米), Basalt (O).

Figure 5. $\mathrm{Nb} / \mathrm{Y}$ vs $\mathrm{Zr} / \mathrm{TiO}_{2}$ plot for Nakora volcanics (Winchester and Floyd, 1977) [49].

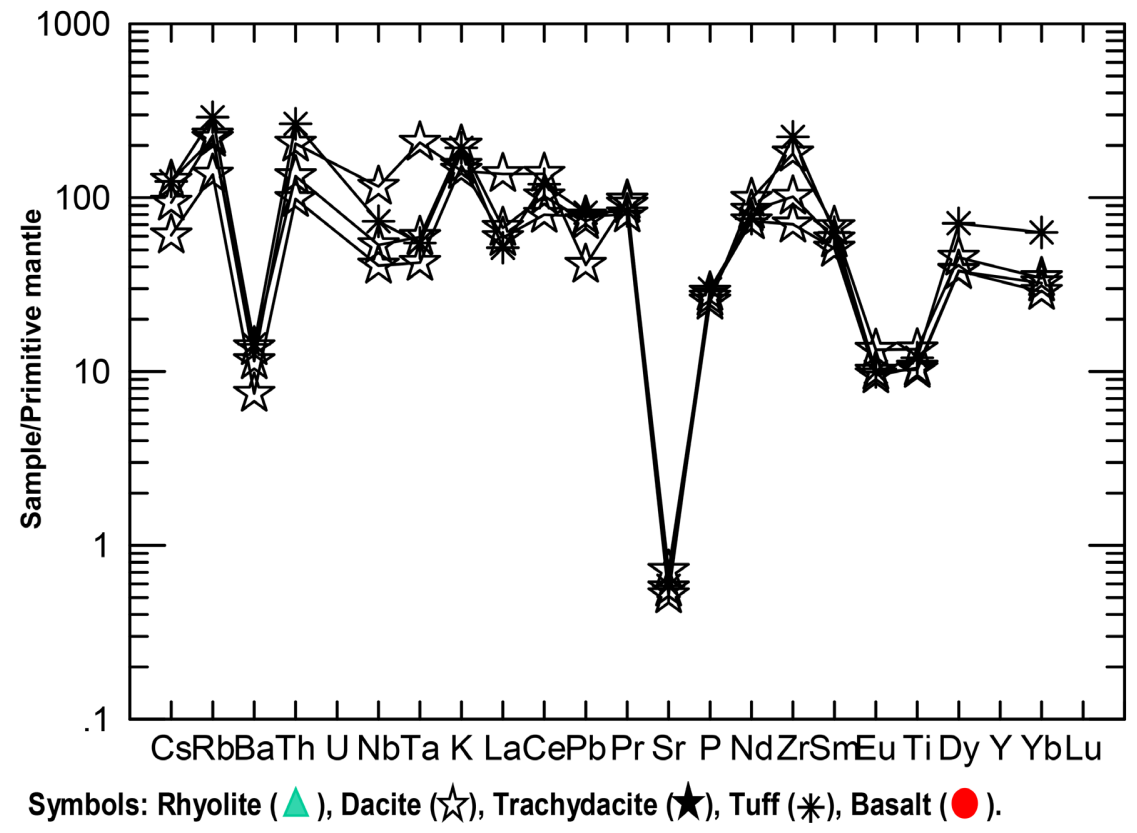

Figure 6. Primitive mantle normalized trace element patterns for selected Nakora acid volcanic rocks (124, 56, $152 \&$ 89) (Sun and McDonough, 1989) [50].

crust (Green 1980) [51].

\section{Chondrite Normalized Patterns of Rare Earth Elements}

The chondrite normalized patterns for acid volcanics are shown in Figure 7 


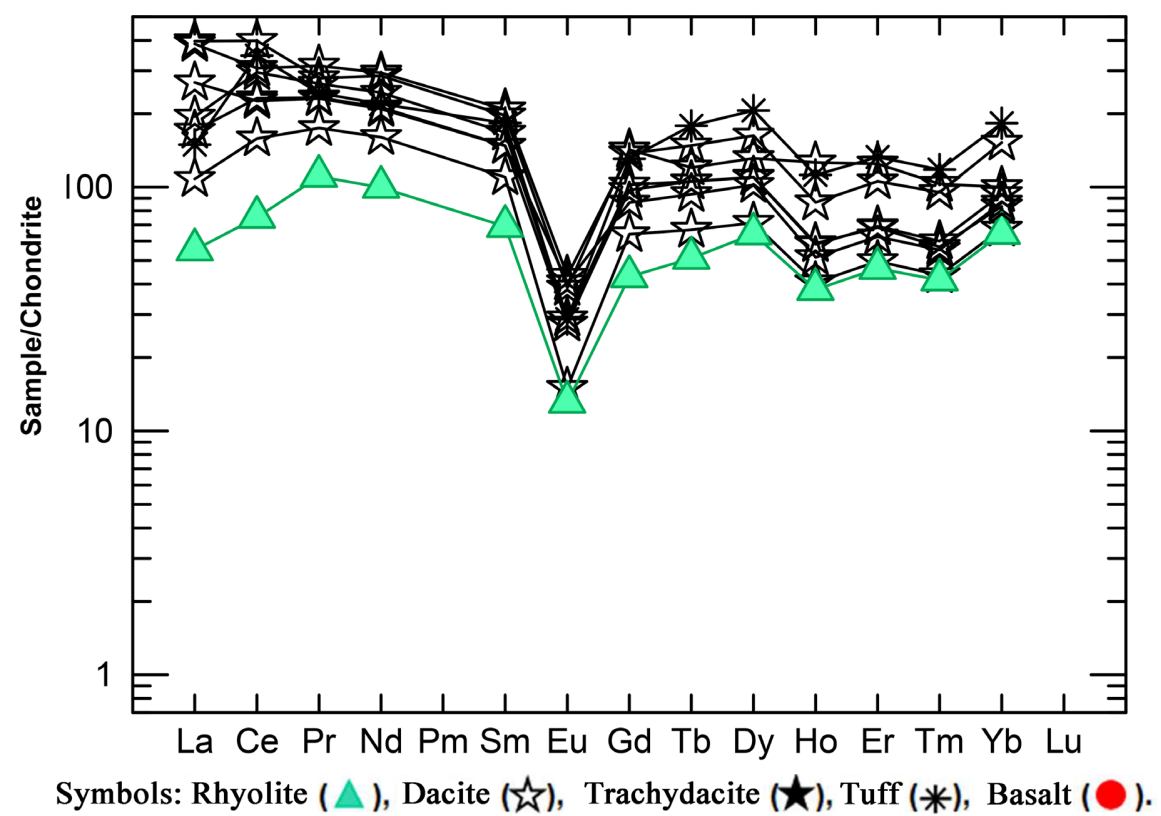

Figure 7. Chondrite normalized REE patterns for Nakora acid rocks $(27,28,164,15,56$, 124, 126 \& 89) (Sun and McDonough, 1989) [50].

(normalization values after Sun and McDonough (1989) [50]. The Nakora acid volcanics show higher concentrations of flat LREE with increasing negative anomaly and lower concentration of HREE. The REE concentration of rhyolite is less as compared to dacite and tuff. The very pronounced negative Eu anomaly indicates strong fractionation and partioning by feldspar. A significant distinguishing feature in REE values is related to the magnitude of Eu anomalies.

REE patterns in the Nakora acid volcanics and basic rocks are characterized by sub-parallel patterns with strong negative $\mathrm{Eu}$ anomaly $\left(\mathrm{Eu} / \mathrm{Eu}^{*}=0.06\right.$ to 0.12 , avg. 0.08 ). But in basic, positive $\mathrm{Eu}$ anomaly ( $\mathrm{Eu} / \mathrm{Eu}^{*}=0.24$ to 0.44 , avg. 0.34$)$ is observed. The Nakora basic rocks are less enriched in LREE and HREE as compared to acid volcanics. On the other hand, rhyolites are showing almost similar abundances of REE which is probably due to their comagmatic nature. In general, the fractionation is more in HREE as compared to LREE in Nakora acid volcanic rocks, whereas in basics, almost flat normalized patterns are observed. Thus the sub-parallel REE patterns of all Nakora rocks suggest a common magmatic source.

\section{Petrogenesis}

The basic rocks of MIS are derived by different degree of partial melting of lithosphere source rocks (alkaline and subalkaline) in Aravalli block (Vallinayagam 2003) [44]. Kundal basic rocks are derived from Fe enriched source with higher Fe/Mg ratio than primitive mantle source (Singh and Vallinayagam 2004) [24]. Siwana basic rocks are formed as a result of low degree partial melting of crustal rocks which are enriched in incompatible elements and Fe under the influence of fluid/melt in the lithosphere (Vallinayagam 2001) [21]. 
Bhilwara area are considered as sources for Nakora basalts. In Figure 8, the chondrite normalized pattern is derived for partial melt by $33 \%$ partial melting leaving a residue $48 \%$ plagioclase, $33 \%$ opx and $19 \%$ cpx which closely approaches the REE patterns of Nakora basalts.

In Figure 9 Nakora gabbros are considered as source. It represents the melt generated by $37 \%$ batch partial melting of the Nakora gabbro leaving a residue consisting of $48 \%$ plagioclase, $33 \%$ opx and $19 \%$ cpx which approaches the REE patterns of Nakora basalts.

Hence the Nakora basalts can be derived from the source similar of Nakora gabbro. Here, Bhilwara mafic metavolcanic which is taken from outside the study area and Nakora gabbros taken from the study area are showing maximum similarities with the REE patterns of the Nakora basalts. Hence, the Nakora basalts could have been derived by different degrees of partial melting of source rock similar to Bhilwara mafic metavolcanic/Nakora gabbros composition.

Malani volcanics (Siwana, Barmer and Korna) are generally associated with the central type of eruptions. Alkalinity is associated with central type of eruptions and increase by the breakdown of alkali pyroxene to alkali amphibole by

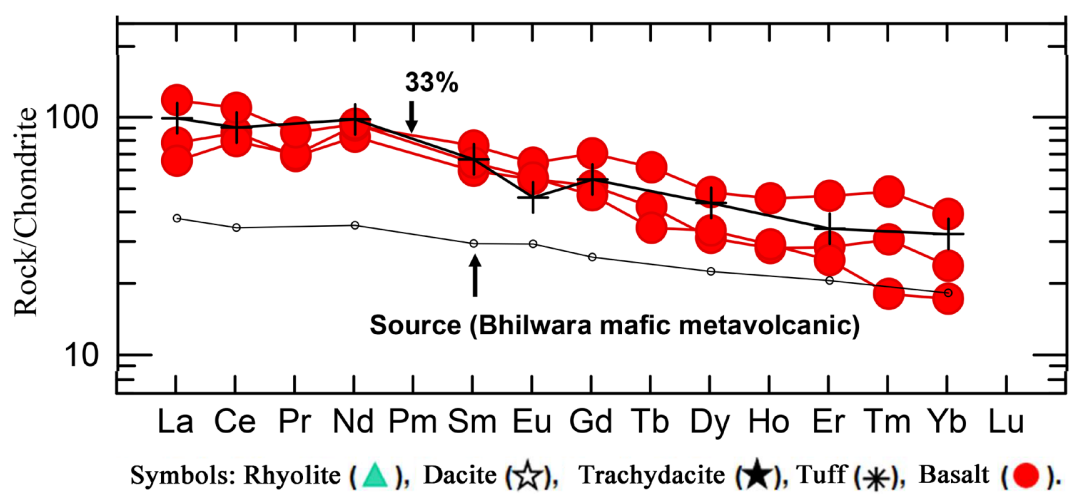

Figure 8. Chondrite normalized diagram showing the calculated REE patterns for melts produced by $33 \%$ batch melting of mafic metavolcanic from Bhilwara leaving a residue consisting of $48 \%$ plagioclase, $33 \%$ opx and $19 \%$ cpx.

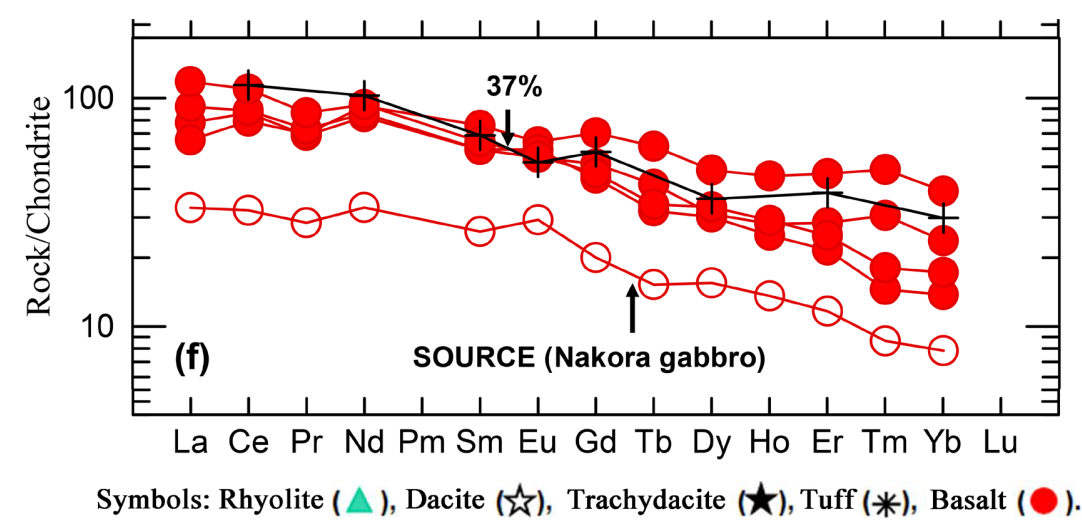

Figure 9. Chondrite normalized diagram showing the calculated REE patterns for melts produced by $37 \%$ batch melting of Nakora gabbro leaving a residue consisting of $48 \%$ plagioclase, $33 \%$ opx and $19 \%$ cpx. 
lowering of $\mathrm{fO}_{2}$ (Bailey 1969) [52]. Silicic magma can be related to partial melting of pre-existing crust, enriched in silica, alumina and alkalies (Mc Burney 1984) [53]. The stability of peralkaline silicates is buffered by arfvedsonite-aegirine equilibrium under NNO buffer conditions (Grapes et al. 1979) [54]. Generally, peralkaline magma exists with non-peralkaline volcanics within a centre and peralkaline rhyolites are produced by late stage fractionation of metaluminous rhyolites (Ewart 1982) [55]. In REE modeling of Nakora acid volcanic rocks, normalization values are taken from Sun and McDonough (1989) [50] and the calculations were made using the mineral/melt partition coefficients of Arth (1976) [56]; Arth and Barker (1976) [57]; Fujimaki (1984) [58] and Green and Pearson (1985) [59].

Siwana rhyolite is considered as a similar source rock for REE modeling of Nakora acid volcanics. The calculated melt is derived by $30 \%$ batch partial melting of Siwana rhyolite leaving a residue consisting of $45 \%$ alkali feldspar, $40 \%$ quartz, $10 \%$ plagioclase and 5\% cpx (Figure 10). The calculated REE pattern of source rock is similar to REE pattern of Nakora acid volcanics. Hence, Nakora acid volcanic rocks could have been generated from a source similar to Siwana rhyolite.

In Figure 11, the calculated REE patterns for 25\% partial melting of banded

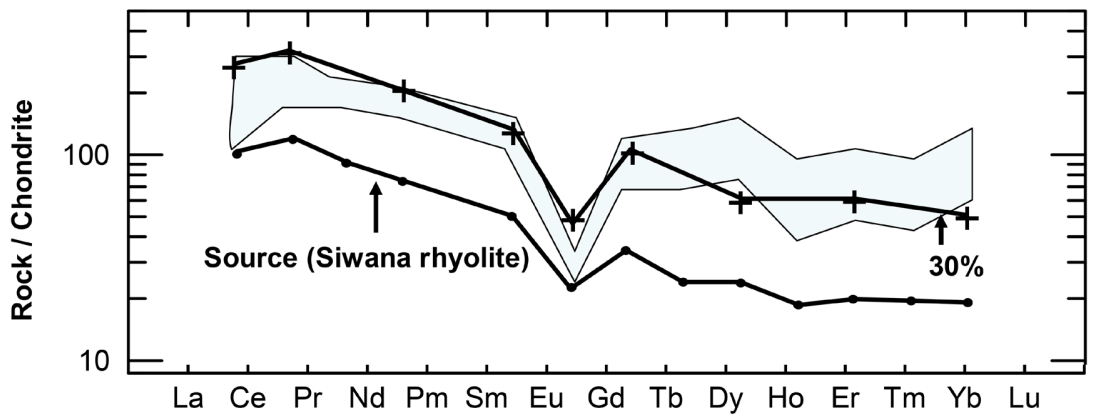

Figure 10. Chondrite normalized diagram showing the calculated REE patterns for melts produced by $30 \%$ batch partial melting of Siwana rhyolite leaving a residue consisting of $45 \%$ alkali feldspar, $40 \%$ quartz and $10 \%$ plagioclase and 5\% cpx. The REE abundances of the Nakora acid volcanics (shaded zone of rhyolites and trachytes) are comparable to that of magma derived from the source.

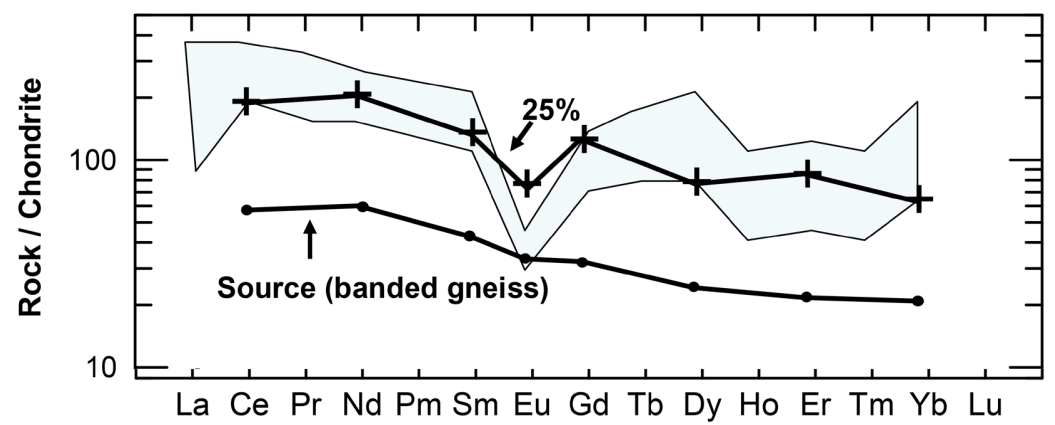

Figure 11. Chondrite normalized diagram showing the calculated REE patterns of Nakora acid rocks (shaded zone) for melts produced by $25 \%$ batch partial melting of banded gneiss leaving a residue consisting of $45 \%$ alkali feldspar, $40 \%$ quartz and $10 \%$ plagioclase and $5 \%$ cpx. 
gneiss from Kolar Schist Belt and 35\% partial melting of Delhi granite is considered. The source sample is leaving a residue consisting of $45 \%$ alkali feldspar, $40 \%$ quartz, $10 \%$ plagioclase and 5\% cpx. REE pattern of banded gneiss is showing more similarities with REE patterns of Nakora acid volcanic rocks. After the Siwana rhyolite, banded gneiss may be the source rock for Nakora acid volcanics.

\section{Conclusions}

Nakora basalts have transitioned from basalt to basaltic andesite. The Nakora basalts show the close affinity to continental field in the $\mathrm{MgO}-\mathrm{Fe}_{2} \mathrm{O}_{3}-\mathrm{Al}_{2} \mathrm{O}_{3}$ diagram (Pearce et al. 1977) [60]. As compared to trachytes, the rhyolites show high $\mathrm{SiO}_{2}$, high $\mathrm{Al}_{2} \mathrm{O}_{3}$, low total alkalies, low total iron, low $\mathrm{TiO}_{2}$, high $\mathrm{CaO}$ and high $\mathrm{MgO}$. The presence of acmite indicates the alkali nature of rhyolites. As compared to rhyolites, the tuff shows low $\mathrm{SiO}_{2}$, high $\mathrm{Al}_{2} \mathrm{O}_{3}$, low total alkalies, low total iron, high $\mathrm{TiO}_{2}$, low $\mathrm{CaO}$ and low $\mathrm{MgO}$.

In the trace and REE geochemical studies, Nakora basalts show high $\mathrm{Cu}, \mathrm{Zn}$, $\mathrm{Rb}, \mathrm{Y}, \mathrm{Zr}, \mathrm{Th}, \mathrm{U}, \mathrm{La}, \mathrm{Ce}, \mathrm{Eu}$ and $\mathrm{Yb}$ and low content of $\mathrm{Ni}$ and $\mathrm{Sr}$. In the primitive mantle normalized diagram of trace elements, Nakora basic rocks show LREE enriched nature with negative $\mathrm{Nb}, \mathrm{Ta}, \mathrm{Sr}$ and $\mathrm{Zr}$ anomalies. As compared to trachyte, rhyolites show low ranges of $\mathrm{Ni}, \mathrm{Cu}, \mathrm{Zn}, \mathrm{Rb}, \mathrm{La}$ and $\mathrm{Ce}$ and high contents of Sr, Y, Zr, Th, U, Nd, Sm, Eu and Yb. Tuff shows low concentrations of $\mathrm{Ni}, \mathrm{Cu}, \mathrm{Zn}, \mathrm{Sr}, \mathrm{La}$ and $\mathrm{Ce}$ and high value of Rb, Y, Zr, Th, U, Nd, Sm, Eu and $\mathrm{Yb}$ than rhyolites. Higher concentrations of LREE and lower concentration of HREE are observed in Nakora acid volcanics. Nakora acid volcanics are enriched in $\mathrm{Rb}, \mathrm{Th}, \mathrm{K}$ and $\mathrm{Zr}$. The low $\mathrm{Sr}$ in Nakora acid volcanics may be due to plagioclase fractionation and $\mathrm{Zr}$ enrichment in the source indicates the original alkaline nature of the magma.

Petrominerolgical and geochemical data suggest that the rocks of NRC are formed from comagmatic source by cogenetic process in rift tectonic setting. Petrogenetic modeling studies indicate that the Nakora basic rocks may be derived from a rock similar to Bhilwara mafic metavolcanic/mixed Nakora gabbros by different degrees of partial melting. Nakora acid volcanic rocks could have been derived by different degree of partial melting of source rock similar to Siwana rhyolite/banded gneiss from Kolar Schist Belt.

The acid volcanic (rhyolites and trachytes) flows are dispersed after the eruption of magma through a volcanic vent in NRC. Basalt-trachyte-rhyolite association suggests that the large amount of heat is supplied to the crust from the magma chamber before the eruption. MIS is characterized by within plate environment which is related to hot spot activity and represents the tensional environment in their emplacement as indicated by various elliptical/ring structures. Trans-Aravalli block of the Indian shield shows the similarities with Central Iran, Nubian-Arabian shield, Medagascar, South China, Somalia and Seychelles in terms of intraplate, mantle plume, crust-mantle interaction, anorogenic na- 
ture and extensional tectonic setting. Hence, mantle plume and crust-mantle interaction can play an important role in understanding the genesis of MIS which should be studied in the future.

\section{Acknowledgements}

The authors are thankful to Late Prof. G. Vallinayagam, Former Head, Department of Geology, Kurukshetra University, Kurukshetra, for his support and guidance. The authors express sincere gratitude to all the people who knowingly and unknowingly supported during this work period.

\section{Conflicts of Interest}

The authors declare no conflicts of interest regarding the publication of this paper.

\section{References}

[1] Kochhar, N. (1973) Indo-Gangetic Basin, Ring Structure and Continental Drift. Nature (London), 242, 141-142. https://doi.org/10.1038/physci242141a0

[2] Pareek, H.S. (1981) Petrochemistry and Petrogenesis of the Malani Igneous Suite, India. GSA Bulletin, 92, 206-273. https://doi.org/10.1130/GSAB-P2-92-206

[3] Kochhar, N. (1984) Tusham Ring Complex, Bhiwani District, India. Proceedings of the Indian National Science Academy, 49, 459-490.

[4] Bhushan, S.K. (1985) Malani Volcanism in Western Rajasthan. Indian Journal of Earth Sciences, 12, 58-71.

[5] Bhushan, S.K. and Mohanty, M. (1988) Mechanics of Intrusion and Geochemistry of Alkaline Granites from Siwana, Barmer District, Rajasthan. Indian Journal of Earth Sciences, 16, 103-115.

[6] Vallinayagam, G. (1988) Geology and Geochemistry of Alkali Granites and the Associated Acid Volcanics around Mokalsar, District Barmer, W. Rajasthan, India and Their Bearing on the Rift Tectonics. Unpublished Ph.D. Thesis, Punjab University, Chandigarh, 1-113.

[7] Bhushan, S.K. (1989) Mineral Chemistry and Petrogenetic Aspects of Malani Volcanics, Western Rajasthan. Indian Minerals, 43, 325-338.

[8] Eby, G.N. and Kochhar, N. (1990) Geochemistry and Petrogenesis of the Malani Igneous Suite, North Peninsular India. Journal of the Geological Society of India, 36, $109-130$

[9] Baskar, R. (1992) Petrology and Geochemistry of the Alkali Granites and the Associated Acid Volcanics around Goliya Bhalyan, District Barmer, W. Rajasthan, India. Unpublished Ph.D. Thesis, Punjab University, Chandigarh, 153.

[10] Kochhar, N. and Dhar, S. (1993) The Association of Hypersolvus Subsolvus Granites: A Study of Malani Igneous Suite, India. Journal of the Geological Society of India, 42, 449-467.

[11] Baskar, R. and Sharma, S. (1994) An Assessment of the Rare Metal Potential of the Granitoids of Siwana, Jalor, Jhunjhunu and Tosham, North Western Peninsular India. Current Science, 66, 67-69.

[12] Sharma, R. (1994) High Heat Production (HHP) Granites of Jhunjhunu Area, Rajasthan, India. Bulletin of the Indian Geologists Association, 27, 55-61. 
[13] Kochhar, N., Dhar, S. and Sharma, R. (1995) Geochemistry and Tectonic Significance of Acid and Basic Dykes Associated with Jalor Magmatism, Western Rajasthan. Memoir Geological Society of India, 33, 375-389.

[14] Dhar, S., Frel, R., Kramer, S.J.D., et al. (1996) Sr, Pb and Nd Isotope Studies and Their Bearing on the Petrogenesis of the Jalor and Siwana Complexes, Rajasthan, India. Journal of the Geological Society of India, 48, 151-160.

[15] Vallinayagam, G. (1997) Minerals Chemistry of Siwana Ring Complex, W. Rajasthan, India. The Indian Mineralogist, 31, 37-47.

[16] Bhushan, S.K. and Chittora, V.K. (1999) Late Proterozoic Bimodal Volcanic Assemblage of Siwana Subsidence Structure, Western Rajasthan. Journal of the Geological Society of India, 53, 433-452.

[17] Vallinayagam, G. (1999) Nb, Zr, REE Rich Acid Dyke Rocks from the Piplun Area, Siwana Ring Complex, Western Rajasthan, India. In: Paliwal, B.S., Ed., Geological Evolution of Northwestern India, Scientific Publishers, Jodhpur, 94-102.

[18] Bhushan, S.K. (2000) Malani Rhyolites: A Review. Gondwana Research, 3, 65-77. https://doi.org/10.1016/S1342-937X(05)70058-7

[19] Pandit, M.K., Ashwal, L.D., Tucker, R.D., et al. (2001) Proterozoic Acid Magmatism in the Northwestern Indian Shield and Its Significance for Rodinia Construction. Gondwana Research, 4, 726-728. https://doi.org/10.1016/S1342-937X(05)70522-0

[20] Torsvik, T.H., Carter, L.M., Ashwal, L.D., et al. (2001) Rodinia Refined or Obscured: Palaeomagnetism of the Malani Igneous Suite (NW India). Precambrian Research, 108, 319-333. https://doi.org/10.1016/S0301-9268(01)00139-5

[21] Vallinayagam, G. (2001) Geochemistry and Petrogenesis of Basic Rocks in the Siwana Ring Complex, Barmer District, Rajasthan, India. Indian Minerals, 35, 121-133.

[22] Bhushan, S.K. and Chandrasekaran, V. (2002) Geology and Geochemistry of the Magmatic Rocks of the Malani Igneous Suite and Tertiary Alkaline Province of Western Rajasthan. Memoirs of Geological Survey of India, Vol. 126, Geological Survey of India, Kolkata, 1-129.

[23] Kochhar, N. (2004) Geological Evolution of the Trans-Aravalli Block (TAB) of the NW Indian Shield: Constraints from the Malani Igneous Suite (MIS) and Its Seychelles Connection during Late Proterozoic. Special Publication of Geological Survey of India, 84, 247-264.

[24] Singh, A.K. and Vallinayagam, G. (2004) Geochemistry and Petrogenesis of Anorogenic Basic Volcanic-Plutonic Rocks of the Kundal Area of Malani Igneous Suite, Western Rajasthan, India. Journal of Earth System Science, 113, 667-681. https://doi.org/10.1007/BF02704028

[25] Vallinayagam, G. (2004) A Report on Rare Metals and Rare Earths in the Siwana Ring Complex, Rajasthan. Applied Geochemistry, 6, 387-391.

[26] Bhushan, S.K. and Chittora, V.K. (2005) Proterozoic Granitoids of Rajasthan. Journal of the Geological Society of India, 66, 741-763.

[27] Singh, A.K., Singh, R.K.B. and Vallinayagam, G. (2006) Anorogenic Acid Volcanic Rocks in the Kundal Area of the Malani Igneous Suite, Northwestern India: Geochemical and Petrogenetic Studies. Journal of Asian Earth Sciences, 27, 544-557. https://doi.org/10.1016/j.jseaes.2005.05.008

[28] Vallinayagam, G. and Kumar, N. (2007) Volcanic Vent in Nakora Ring Complex of Malani Igneous Suite, Northwestern India. Journal of the Geological Society of India, 70, 881-883.

[29] Vallinayagam, G. and Kumar, N. (2008) Flow Stratigraphy of Nakora Ring Com- 
plex, Malani Igneous Suite, Rajasthan, NW Peninsular India. Journal of the Geological Society of India, 91, 127-135.

[30] Kochhar, N. (2009) The Malani Supercontinent: Middle East Connection during Late Proterozoic. Economic Mineralization (ed. Shrivastava) Scientific Publishers (India), Jodhpur, 15-25.

[31] Singh, A.K. and Vallinayagam, G. (2009) Radioactive Element Distribution and Rare-Metal Mineralization in Anorogenic Acid Volcano-Plutonic Rocks of the Neoproterozoic Malani Felsic Province, Western Peninsular India. Journal of the Geological Society of India, 73, 837-853. https://doi.org/10.1007/s12594-009-0067-Z

[32] Vallinayagam, G. (2009) Occurrence of Zinc Rich A-Type Granites in the Trans-Aravalli Anorogenic Ring Complexes, Northwestern India. In: Shrivastava, K.L., Ed., Economic Mineralization, Scientific Publishers, Jodhpur, 205-209.

[33] Kumar, N. and Kumar, N. (2020) Geochemistry of Volcanic Flows of Nakora Area of Malani Igneous Suite, Northwestern India: Constraints on Magmatic Evolution and Petrogenesis. International Journal of Engineering, Science and Technology, 12, 66-82. https://doi.org/10.4314/ijest.v12i1.6

[34] Qasim Jan, M., Lachari, A. and Asif, K. (1997) Petrography of the Nagar Parkar Igneous Complex, Tharparkar, SE Sindh. Geological Bulletin, University of Peshawar, 30, 227-249.

[35] Blanford, W.T. (1877) Geological Notes on the Great Indian Desert between Sind and Rajasthan. Records of the Geological Survey of India, 10, 10-21.

[36] La Touche, T.H.D. (1902) Geology of Western Rajputana. Memoirs of the Geological Survey of India, 35, 1-116.

[37] Pascoe, E.H. (1959) A Manual of the Geology of India and Burma. 2nd Edition, Government of India, Delhi, 448 p.

[38] Mukherjee, A.B. (1962) Pantelleritic Assemblages from W. Rajasthan. Quarterly Journal Geological, Mining and Metallurgical Society, India, 34, 149.

[39] Kochhar, N., Vallinayagam, G. and Gupta, L.N. (1991) Zircon from the Granitic Rocks of the Malani Igneous Suite: Morphological and Chemical Studies. Journal of the Geological Society of India, 38, 556-576.

[40] Vallinayagam, G. and Kochhar, N. (1998) Geochemical Characterization and Petrogenesis of A-Type, Granites and the Associated Acid Volcanics of the Siwana Ring Complex, Northern Peninsular, India. In: Paliwal, B.S., Ed., The Indian Precambrian, Scientific Publishers, Jodhpur, 460-481.

[41] Kochhar, N. (2000) Attributes and Significance of the A-Type Malani Magmatism, Northwestern Peninsular India. In: Deb, M., Ed., Crustal Evolution and Metallogeny in the Northwestern India Shield, Narosa Publishing House, New Delhi, 158-188.

[42] Sharma, R. and Kumar, N. (2017) Petrology and Geochemistry of A-Type Granites from Khanak and Devsar Areas of Bhiwani District, Southwestern Haryana. Journal of the Geological Society of India, 90, 138-146. https://doi.org/10.1007/s12594-017-0691-y

[43] Kumar, N., Kumar, N. and Singh, A.K. (2019) Petrology and Geochemistry of Acid Volcano-Plutonic Rocks from Riwasa and Nigana Areas of Neoproterozoic Malani Igneous Suite, Northwestern Peninsular India: An Understanding Approach to Magmatic Evolution. Geochemistry International, 57, 645-667. https://doi.org/10.1134/S001670291906003X

[44] Vallinayagam, G. (2003) Basic Magmatism of Neoproterozoic Malani Igneous Suite, 
Western Indian Craton: Petrological and Geochemical Modelling. Indian Journal of Geochemistry, 18, 1-18.

[45] Kochhar, N. (1984) Malani Igneous Suite: Hot-Spot Magmatism and Cratonization of the Northern Part of the Indian Shield. Journal of Geological Society of India, 25, 155-161.

[46] Shapiro, L. and Brannock, W.W. (1962) Rapid Analysis of Silicates, Carbonates and Phosphate Rocks. U.S. Geological Survey Bulletin 1144A, 1-56.

[47] Giret, A., Bonin, B. and Leger, J.M. (1980) Amphibole Compositional Trends in Oversaturated and Undersaturated Alkaline Plutonic Ring-Complexes. The Canadian Mineralogist, 18, 481-495.

[48] Le Bas, M.J., Maitre, R.W., Streckeisen, A., et al. (1986) A Chemical Classification of Volcanic Rocks Based on the Total Alkali Silica Diagram. Journal of Petrology, 27, 745-750. https://doi.org/10.1093/petrology/27.3.745

[49] Winchester, J.A. and Floyd, P.A. (1977) Geochemical Discrimination of Different Magma Series and Their Differentiation Products Using Immobile Elements. Chemical Geology, 20, 325-343. https://doi.org/10.1016/0009-2541(77)90057-2

[50] Sun, S.S. and Mc Donough, W.F. (1989) Chemical and Isotopic Systematics of Oceanic Basalts: Implications for Mantle Composition and Processes. Geological Society, London, Special Publications, 42, 313-345.

https://doi.org/10.1144/GSL.SP.1989.042.01.19

[51] Green, T.H. (1980) Island Arc and Continent Building Magmatism: A Review of Petrogenetic Models Based on Experimental Petrology and Geochemistry. Tectonophysics, 63, 367-385. https://doi.org/10.1016/0040-1951(80)90121-3

[52] Bailey, D.K. (1969) The Stability of Acmite in the Presence of $\mathrm{H}_{2} \mathrm{O}$. American Journal of Science, 267, 1-16. https://doi.org/10.2475/ajs.267.2.242

[53] Mc Burney, A.R. (1984) Igneous Petrology. Freeman Cooper and Co., San Francisco, $378 \mathrm{p}$.

[54] Grapes, R., Yagi, K. and Okumura, K. (1979) Aenigmatite, Sodic Pyroxene, Arfvedsonite and Associated Minerals in Syenites from Morotu, Sakhalin. Contributions to Mineralogy and Petrology, 69, 97-103. https://doi.org/10.1007/BF00371853

[55] Ewart, E. (1982) Petrogenesis of the Tertiary Anorogenic Volcanic Series of Southern Queensland, Australia, in the Light of Trace Element Geochemistry and O, Sr and $\mathrm{Pb}$ Isotopes. Journal of Petrology, 23, 344-382. https://doi.org/10.1093/petrology/23.3.344

[56] Arth, J.G. (1976) Behaviour of Trace Elements during Magmatic Process Summary of Theoretical Models and Their Applications. Revised Geophysical Space Physics, 15, 96-104.

[57] Arth, J.G. and Barker, F. (1976) Rare Earth Partitioning between Hornblende and Dacitic Liquid Implication for the Genesis of Trondhjemitic-Tonalitic Magmas. Geology, 4, 534-536. https://doi.org/10.1130/0091-7613(1976)4<534:RPBHAD>2.0.CO;2

[58] Fujimaki, H. (1984) Partition Coefficients of Hf, Zr and REE between Phenocrysts and Groundmass. Journal of Geophysical Research, 89, 662-672. https://doi.org/10.1029/JB089iS02p0B662

[59] Green, T.H. and Pearson, N.J. (1985) Rare Earth Partitioning between Clinopyroxene and Silicate Liquid at Moderate to High Pressure. Contributions to Mineralogy and Petrology, 91, 24-36. https://doi.org/10.1007/BF00429424

[60] Pearce, T.H., Gorman, B.E. and Birkett, T.C. (1977) The Relationships between 
Major Element Chemistry and Tectonic Environment of Basic and Intermediate Volcanic Rocks. Letters of Earth Planetary Science, 36, 121-132.

https://doi.org/10.1016/0012-821X(77)90193-5 